\title{
Re: Impact of the availability of midurethral slings on treatment strategies for stress urinary incontinence: a cost-effectiveness analysis
}

\author{
MANVI VERMA ${ }^{1}$ and Jaya Chaturvedi ${ }^{1}$ \\ ${ }^{1}$ All India Institute of Medical Sciences - Rishikesh
}

February 23, 2022

Dr Manvi Verma (ORCID ID: 0000-0001-5971-2940)

Article Type: Letter to the Editor

"Re: Impact of the availability of midurethral slings on treatment strategies for stress urinary incontinence: a cost-effectiveness analysis"

Dr Manvi Verma ${ }^{1}$, Dr Jaya Chaturvedi ${ }^{2}$

M.Ch. Student in Cosmetic Gynaecology, Department of Obstetrics \& Gynaecology, All India Institute of Medical Sciences, Rishikesh, Uttarakhand, India

Professor \& Head, Department of Obstetrics \& Gynaecology, All India Institute of Medical Sciences, Rishikesh, Uttarakhand, India

\section{Corresponding Author:}

Dr Manvi Verma, MD, DNB

Department of Obstetrics \& Gynaecology, All India Institute of Medical Sciences, Rishikesh, Uttarakhand, India, 249201

manvi278@gmail.com

$+919469388553$

Short Title: Cost-effectiveness analysis of stress incontinence treatment

Dear Editor,

We commend Chang et al for their elaborate work on analyzing the cost-effectiveness of surgical and nonsurgical treatment strategies for stress urinary incontinence. ${ }^{1}$ The authors modeled seven pathways for the treatment of stress urinary incontinence and found out that incontinence pessary, pelvic floor muscle physical therapy, and midurethral slings were the only cost-effective treatment options. Amongst non-surgical treatment modalities, pelvic floor muscle therapy is the most cost-effective strategy. ${ }^{1,4}$

The analysis done by the authors has added a much-needed boost to finding cost-effective solutions for women with stress urinary incontinence and may have implications for improving patient care and decreasing financial toxicity. It is commendable that surgeons from geographically diverse institutions in the United States have developed treatment plans for individual pathways. Also, the time duration has been set to two years which is also absolutely logical as maximum complications and retreatments will occur within this 
period. We feel that the outcomes measured (Incremental Cost-Effectiveness Ratio and Quality-AdjustedLife-Years) by the author are appropriate and can help in better policy formulation.

In nations with resource constraints like India, general medical insurance schemes like Ayushman Bharat have provided a silver lining to the common man. However, when the cost of the implant is not covered under such General Insurance Cover (GIC), patients are forced to opt for a low-quality alternative option with less durability. In such cases, the role of finding cost-effective solutions comes into the picture as affordable solutions need to be quality controlled and regulated under minimum specific standards, otherwise, they may lead to poor surgical results in the long run. Midurethral slings have been found as the most cost-effective surgical option unanimously. ${ }^{1-3}$ We suggest that the material of the mesh also needs to be included in such cost-effectiveness analysis and long-term comparative trials be conducted on the same subject.

We believe that the inherent limitation of retrospective studies for such cost-effectiveness analysis is that, they can miss many additional real-life costs incurred by the patients as the data has been collected from medical reimbursement data or published literature. While planning a surgery for stress urinary incontinence factors like cost of consumables, complications, re-treatments, complementary tests, treatments, and consultations that would be carried out before and after each surgery also play a role. ${ }^{3}$ Also, COVID-19 has made an unprecedented impact on the affordability of common masses where the quality-of-life health issues like stress urinary incontinence have taken the backburner.

We suggest that prospective multi-center trials should be conducted to assess the real economic ramifications and the holistic expenses incurred by patients. This will help us move towards a better understanding and crowd-sourcing of solutions to provide cost-effective health care services to women with stress urinary incontinence, which affects almost one-third of the female population in India, similar to USA.

Funding/Financial support: No funding was received for this study

Acknowledgements : None

Disclosure of interests : The authors report no conflict of interest

\section{Contributions to authorship:}

MV - Searched the literature, prepared the manuscript, drafted it and revised it

JC- Revised the manuscript

References

1. Chang OH, Cadish LA, Kailasam A, Ridgeway BM, Shepherd JP. Impact of the availability of midurethral slings on treatment strategies for stress urinary incontinence: a cost-effectiveness analysis. BJOG. 2022 Feb;129(3):500-508.

2. Seklehner S et al. A Cost-Effectiveness Analysis of Retropubic Midurethral Sling Versus Transobturator Midurethral Sling for Female Stress Urinary Incontinence. Neurourology and Urodynamics. 2014; 33:1186-1192.

3. Javanbakht $\mathrm{M}$ et al. Economic evaluation of surgical treatments for women with stress urinary incontinence: a cost-utility and value of information analysis. BMJ Open 2020;10: e035555.

4. Simpson AN et al. A Cost-Utility Analysis of Nonsurgical Treatments for Stress Urinary Incontinence in Women. Female Pelvic Med Reconstr Surg. 2019 Jan/Feb;25(1):49-55. 